\title{
ADHESIONAL INSTABILITIES and GECKO LOCOMOTION
}

\author{
John A Williams* \\ Cambridge University Engineering Department, \\ Trumpington Street, Cambridge, CB2 1PZ, UK.
}

\begin{abstract}
Geckos possess a remarkable ability to run rapidly on both walls and ceilings and in recent years the mechanisms that underlie this facility have come under close scrutiny. It is now generally agreed that one of the principal mechanisms of adhesion relies on the action of van der Waal forces acting between the final extremely fine structure of the gecko toe and the underlying substrate. High speed video analysis shows that adhesive contact is both made and broken in intervals of less than $20 \mathrm{~ms}$ and this suggests that the mechanism of detachment is one of adhesive instability rather than steady-state peeling. By considering the gecko seta/spatula as a Euler-Bernoulli cantilever it is possible to model this instability in non-dimensional terms and thus to test the analysis at a much larger scale with more conventional engineering materials. When applied to the scale and material combination appropriate to a gecko spatula the predicted critical load, of around $10 \mathrm{nN}$, is close to values that have been observed using and AFM cantilever and a single detached spatula.
\end{abstract}

Keywords: adhesion, gecko, instability

*jaw@eng.cam.ac.uk 


\section{NOMENCLATURE}

\begin{tabular}{llll}
$b$ & width of flexible laminate & $P$ & force \\
$w$ & work of adhesion per unit area & $E$ & elastic modulus \\
$h$ & thickness & $I$ & second moment of area \\
$\ell$ & length & $M$ & bending moment \\
$d$ & diameter & $M_{0}$ & critical value of bending moment \\
$\theta$ & peel angle & $\mathbf{M}$ & non-dimensional moment $M_{0} / \sqrt{2 b w E I}$ \\
$\mathbf{P}$ & non-dimensional load $P / b w$ & $\alpha$ & $\arcsin ((1+\mathbf{P}) / 2 \mathbf{P})$ \\
$\mathbf{L}$ & non-dimensional length $\ell \sqrt{2 b w / E I}$ & $\beta$ & $\arcsin (\mathbf{P} /(1+\mathbf{P}))$ \\
$\phi$ & cantilever tip rotation & $\phi_{t}$ & $\operatorname{spatula~tip~rotation}$ \\
$U$ & stored elastic energy & $\mathbf{K}(\alpha), \mathbf{F}(\beta \backslash \alpha)$ elliptical integrals \\
$\mathbf{1}$ & INTRODUCTION & \multicolumn{2}{l}{}
\end{tabular}

Over the past fifteen years or so the fibrillar attachment systems of insects, arachnids and reptiles have been intensively studied with the aim of both understanding their extraordinary success and exploring the possibilities of mimicing their performance with artificial structures and materials. It is now generally agreed that an essential mechanism of dry adhesion for all these creatures depends on the action of van der Waal forces acting between the final physiological structure of the animal and the surface on which it moves. In humid atmospheres there may be a thin layer of water on both surfaces which will attenuate this effect: on the other hand, if there is sufficient water to form micro-menisci then the associated Laplace pressure drops may also contribute positively to adhesion [Riedo et al 2002].

The heaviest creatures capable of climbing and manoeuvring on smooth vertical or inverted surfaces are geckos and consequently the morphology of their attachment system has come under particularly close scrutiny. The structural hierarchy of the toe structure of the Tokay gecko (Gekko gecko) is illustrated in Fig. 1 [Autumn, Liang et al 2000, Gao et al 2005, Tian, Pesika et al 2006]. At the first level, each toe possesses a series of ridges or lamellae which consist of a mass of curved keratin hairs, the setae. These are typically about $100 \mu \mathrm{m}$ long and 5-10 $\mu \mathrm{m}$ diameter and are closely packed to a density of more than 
Revised -15 October 2014 $10,000 \mathrm{~mm}^{-2}$. Although the modulus of keratin is high, in excess of $1 \mathrm{GPa}$ [ Peattie, Majidi et al 2007], this fibrous structure provides a compliant pad with an effective modulus of only ca. $100 \mathrm{kPa}$ [Autumn, Majidi et al 2006] so that there can be greatly improved conformation between the contours of the gecko's toes and the surface with which they are in contact. However, in order to generate successful macro-adhesion through the action of van der Waal forces, which have a range of only fractions of a nanometre, even closer and more intimate contact is necessary. This is achieved through the multiple division of each seta at its end into a branched structure terminating in a multitude of slightly angled and flattened spatulae each of which is only perhaps $200 \mathrm{~nm}$ wide and only $5 \mathrm{~nm}$ in thickness. The shaft of the spatula from its point of branching with the much more robust seta to the point at which it flattens out is typically less than $1 \mu \mathrm{m}$ long and perhaps about $100 \mathrm{~nm}$ in diameter [ Tian et al 2006]. This hierarchy enables the 'true' area of contact between toe and substrate to approach a significant proportion of the geometric or nominal area and this allows the effect of adhesion to be macroscopically significant to such an extent that it has been estimated that if all of the spatulae were optimally employed each foot of a gecko could generate adhesion of ca. $100 \mathrm{~N}$ [Autumn, Liang et al 2000].

Almost equal in importance to the ability to generate adequate adhesion is the ability to release the bond in a controlled yet rapid fashion. It is possible to draw a parallel between the way a gecko spatula loses contact during locomotion and the peeling of a flexible tape from a rigid surface by a tensile force applied at its distant end. For the geometry illustrated in Fig. 2(a) it is straightforward [Kendall 1975a] to show that that the tension $P$ in the tape required to bring about detachment is related to its width $b$, elastic modulus $E$, and the peel angle $\theta$ by the relation

$$
(P / b)^{2}(1 / 2 h E)+(P / b)(1-\cos \theta)=w
$$

where $w$ is the work of adhesion that is the energy required to de-cohere unit area of the interface at the peel rate concerned. This equation can be derived by considering the energy balance between the elastic energy within the element of tape released, the work done by the peeling agent and the surface energy change as the junction separates. In the biological context, the value of $w$ is determined by the type and strength of molecular interactions for the materials concerned - in the case of the gecko these will therefore be 
keratin and the somewhat arbitrary nature of the natural substrate and thus beyond the creature's control. On the other hand, the peel angle $\theta$ is a parameter whose value can be controlled and it too has a significant impact on the peel force - reducing $\theta$ increases $P$. It has been demonstrated that control of this parameter during the attachment/detachment cycle plays a key role: setal attachment requires a perpendicular pre-load followed by a small proximal drag, i.e. in a direction towards the animal, which reduces the angle, typically to about $25^{\circ}$, made by the setae with the substrate plane [Gravish, Wilkinson and Autumn 2008]. On the other hand, detachment is accompanied by an increase in the angle made by each setal shaft to about $30^{\circ}$ [Autumn, Dittmore et al 2006].

Returning to the tape analogy, if the material of the tape is very thin and yet of a very high modulus then we may neglect the first term in eqn (1) and so simplify to

$$
P=w b /(1-\cos \theta)
$$

from which it is clear that the peel width $b$ is a purely geometric variable that essentially controls the maximum value of $P$ which can be attained. In principle, in finely divided or hairy adhesive pads, dimension $b$ can grow virtually infinitely, perhaps under evolutionary pressure, to split the attachment elements into smaller and smaller ultimate units. This is illustrated schematically in Fig. 2(b), [after Varenberg, Pugno and Gorb 2010] which shows how the same attachment pad area can become more effective when the overall length of the 'peel line', i.e. the sum of all the participating elements, is increased. In this case a three fold increase in peel line (i.e. $6 \times \frac{1}{2}$ ) for the same overall pad area, increases adhesion by a factor of three. If this hypothesis is correct, namely that potential adhesion scales with total peel line, then we might expect a correlation in nature between total peel line and animal mass across a range of length scales and species. These data have been assembled [Varenberg, Pugno and Gorb 2010] and a linear plot on log/log axes is shown in Fig 3 where there is indeed close correlation between these two quantities (the line shown indicates that peel line $\propto$ mass $^{1.14}$ ). Evaluating the value of $\quad w$ from this plot suggests a value of the order of only $1 \mathrm{~mJ} \mathrm{~m}^{-2}$ which is well below the value of perhaps up to several tens of $\mathrm{mJ} \mathrm{m}^{-2}$ that might be expected for dry organic materials [Israelachvili 1992]. It would seem, under normal circumstances, that each of these creatures is using only a very small percentage of their ultimate adhesive capability. 
While this simple peeling argument referred to above demonstrates the value of dividing the adhesive structure into finer and finer elements, the geometry of Fig. 2, where the tape is peeled by a tensile force aligned with its centre-line, is significantly different from the hierarchical divided beam-like structures evident in Fig. 1 so that the analogy should not be pressed too far. In particular, setae are never perpendicular to the substrate but always make an oblique contact with the seta tips oriented in the distal leg direction [Federle 2006]. This means that when in contact with a substrate the array of setae and their associated spatulae are loaded in bending rather than tension by the combination of the self-weight of the creature and any inertia loads generated by its motion.

\section{MECHANISM of DETACHMENT}

Whole animal adhesion measurements show that detachment occurs effectively spontaneously. It takes barely any longer for the gecko to detach a toe than to bring the toepad into contact - both typically no more than about 15-20 ms [Autumn et al 2006, Gravish, Wilkinson and Autumn 2008]. This suggests that rather than detachment of successive setae happening sequentially there is loss of contact, more or less simultaneously, from a large number of individual spatula contacts. As the animal advances so a multitude of contact points are made in parallel but an equal number are detached at the same rate. Detachment of an adhesive junction requires the injection of energy into the system essentially to satisfy the additional surface energy requirement of the two new free surfaces above that associated with unit area of the single interface. Ultimately this energy must either be supplied by the musculature of the animal aided, perhaps, by gravity if the animal is climbing or hanging.

In the case of the gecko, the adhesive structure has macro-, meso- , micro- and nanofeatures and considerable ingenuity has gone into investigating the mechanics whereby this complex arrangement provides such an effective attachment system, see for example [Bhushan 2007]. The final two scales are critical. Each seta, of which there may be as many as 200,000 per toe, divides into several hundred spatula. In their natural configuration the spatula pads are at an angle of approximately $30^{\circ}$ to the setal shaft and approximately normal to the spatula shaft. When geckos make contact with a substrate they roll their toes inwards [Autumn, Hsieh et al 2006] reducing $\theta_{s}$ the angle that the setal shaft makes with the substrate to perhaps as little as $10^{\circ}$, as illustrated in Fig. 4(b). Since the seta/spatula structure 
has no musculature this must be achieved by manipulating the base of the seta where it is attached to the toes and consequently involves deformation, essentially in bending, of the setal and spatula shafts. In so doing mechanical energy is stored by virtue of the elastic deformation of these components and this energy is subsequently available, released by appropriate changes in the geometry, to contribute to that needed subsequently to separate the adhesive bond. Since this energy is distributed throughout the array it can be made available to a large number of setae and spatulae acting in parallel, i.e. effectively simultaneously, rather than sequentially as would be the case in a quasi-steady-state peeling operation.

Figure 5 illustrates a simplified view of a single seta and its associated $n$ spatulae subject to an end load represented by the load $P$ on each spatula or $n P$ on the whole assembly. Provided the deformation of each of these structural elements is small, i.e. that the tip defection is less than say $10 \%$ of the axial length, then it is relatively straightforward to apportion both deflection and stored elastic energy between the seta and its $n$ spatulae where $n$ might be several hundred. The deflection of seta and individual spatula, measured by the rotation, say $\phi$, from their initially linear axis, say $\phi_{\text {seta }}$ and $\phi_{\text {spatula }}$, can be established from simple linearised cantilever theory whereby if $P$ is the tip load, $\ell$ is length and $E I$ is flexural rigidity then $\phi=P \ell^{3} / 3 E I$. This leads, see Appendix I, to an expression for the ratio of the magnitudes of setal and spatular shaft rotation, as

$$
\phi_{\text {spatula }} / \phi_{\text {seta }} \approx(2 / n)\left(\ell_{1} / \ell_{2}\right)\left(d_{2} / d_{1}\right)^{4}
$$

in which subscripts 1 and 2 refer respectively to spatula and seta and $d$ is the diameter of their assumed circular cross-sections. In a similar fashion, the stored elastic energy of distortion $U$, essentially in bending, of a single seta and its associated $n$ spatulae can be evaluated. The total elastic energy of distortion is divided between the two elements, see Appendix I, in the ratio

$$
U_{\text {spatula }} / U_{\text {seta }} \approx(3 / n)\left(\ell_{1} / \ell_{2}\right)\left(d_{2} / d_{1}\right)^{4}
$$

Several authors have reported careful observations of the dimensions of the structural elements of the gecko pad: for example Tian et al [Tian, Pesika et al 2006] suggest that a typical seta has a length of $120 \mu \mathrm{m}$ and shaft diameter of $4.2 \mu \mathrm{m}$ while the corresponding spatula shaft is $0.8 \mu \mathrm{m}$ long and only $100 \mathrm{~nm}$ in diameter. On this basis, and if $n=500$, 
eqn (3) indicates that it is the flexure of the spatulae that dominates the deflection, $\phi_{\text {spatulae }} / \phi_{\text {seta }} \sim 80$. Similarly, eqn (4) shows that the spatulae account for by far the greater part of the elastic energy; for these figures, $U_{\text {spatulae }} / U_{\text {seta }} \sim 125$.

We can test whether each of these defections is sufficiently small to have confidence in these elementary expressions by considering the static load on a single seta when the animal is at rest. Taking the supported mass to be $0.15 \mathrm{~kg}$ and the total 'toe' area as $400 \mathrm{~mm}^{2}$ provides a nominal static loading pressure of about $3.5 \mathrm{kPa}$ [Gillies et al 2014]. If the density of seta is, say, $10,000 \mathrm{~mm}^{-2}$ then each must support a load of $0.35 \mu \mathrm{N}$. Using the setal dimensions suggested above and taking $E=2 \mathrm{GPa}$ this load would generate a tip deflection of $\sim 7 \mu \mathrm{m}$ which from a structural point of view in a cantilever of length $120 \mu \mathrm{m}$ is sufficiently small for linear beam theory to apply, i.e. for deflection to be proportional to tip load. On the other hand, under less ideal conditions of loading or when in addition to selfweight the toes are loaded by additional thrust or inertial forces an individual seta might well sustain a normal force considerably more than this value - accelerations of around $5 \mathrm{~g}$ might be representative [Vanhooydonck et al 2005]. In these circumstances the consequential tip deflection would no longer be small but a very significant proportion of the setal length so that the situation would be no longer adequately described by the small defection equations of a simple cantilever but rather the non-linear equation of the classical elastica [Love 1927].

Much of the work done in bending the seta and spatulae is stored as elastic energy of distortion within them, so that as the gecko's toes roll out of contact the energy required to break the van der Waals adhesion between spatulae and substrate can come not directly from the musculature remote from the hierarchical attachment pad but from energy stored locally within the structure. Release of the spatulae pads will be expedited if, rather than occurring by steady state peeling, conditions at each spatula reach a point of instability when the peel line accelerates as it is driven by the release of stored energy. Hill et al [2011] have demonstrated that relatively small changes in geometry at the proximal end of the seta can lead to the adhesional conditions at its tip, i.e. in the spatulae, becoming unstable so that they effectively spontaneously detach from the surface thereby enabling the gecko toe to be removed from the substrate no less rapidly than its attachment was made. 
The conditions which will just lead to the peeling away of a bent elastic rod, i.e. a single spatula, adhering to a flat substrate have been explored by a number of authors [e.g. Stewart et al 1987, Oyharcabal and Frisch 2005, Majidi et al 2012]. We consider an elastic rod attracted to a fixed flat surface by a uniform attractive surface force, equivalent to an energy of $w$ per unit area, acted on by a normal tip force which causes some length of the rod to become detached. The freed part of the elastic rod may bend so that equilibrium depends on the balance between adhesive energy and bending energy. The critical condition for detachment of the rod from the substrate can be thought of as dependent on either a critical value of stored elastic energy at the point of detachment or, equivalently, the achievement of a critical curvature of the rod by the local bending moment $M_{0}$. This is effectively the same condition for detachment as used by Stewart, MacLachlan and Calladine [1987] albeit, in their case, at a much finer scale for the detachment of muscle fibres. Detachment will be initiated when

$$
M_{0}=\sqrt{2 E I b w}
$$

in which the second moment of area $I$ refers to the section of the released material, i.e. in the case of the gecko toe to the spatula pad, which is of width $b$. We can consider the moment $M_{0}$ to be generated as the product of a force normal to the surface to which the rod is initially attached and a moment arm which is equal to the original full length of the rod minus any foreshortening which arises from its curvature as is illustrated in Fig. 6. If the rod, i.e. the spatula shaft, which has length $\ell$, were very stiff then the root bending moment is simply $P \ell$ where $P$ is the tip force. But if the shaft bends appreciably under the action of force $P$ then the moment arm is reduced by $\Delta$ to $\ell-\Delta$. If the shaft were too flexible then $P(\ell-\Delta)$ may never reach the critical value of $M_{0}$ in eqn (6) as the reduction in the moment arm more than compensates for the increase in force. In this case loss of contact would be by eventual steady peeling rather than by a bending instability.

It is convenient to write these equation in a non-dimensional form, following [Stewart MacLachlan and Calladine 1987], by defining a normalized force $\mathbf{P}$ as $P / b w$, a normalized length $\mathbf{L}$ as $\ell \sqrt{2 b w / E I}$ and a normalized moment as $\mathbf{M}$ as $M_{0} / \sqrt{2 b w E I}$. It then follows that eqn (6) becomes 


$$
\mathbf{M}=1
$$

and if $\Delta \ll \ell$ then for $\mathbf{M}=1$ it follows that $\mathbf{P L}=2$.

It is possible to make some analytic allowance for the foreshortening effect by noting that, at least to a first approximation, $\Delta \approx 3 \delta^{2} / 5 \ell$ from which it follows that, again for $\mathbf{M}=1$,

$$
\mathbf{P L}\left\{1-\mathbf{L}^{4} \mathbf{P}^{2} / 60\right\}=2
$$

The more general solution, allowing for substantial movement of the point of application of the load, can be deduced from the general equations for an elastica, viz. referring to Fig. 6 in which $s$ is distance from the point of adhesion and $\phi$ is slope,

$$
E I \frac{\mathrm{d} \phi}{\mathrm{d} s}=M_{0}-P x \text { where at } x=0, s=0 \text { and } \phi=0
$$

since $\frac{\mathrm{d} x}{\mathrm{~d} s}=\cos \phi$ the shape of the cantilever is described by the relation

$$
E I \frac{\mathrm{d}^{2} \phi}{\mathrm{d} s^{2}}=-P \cos \phi
$$

The analytic solution to this equation can be written using the introduced groups as

$$
\begin{gathered}
\text { PL }=2[\mathbf{K}(\alpha)-\mathbf{F}(\beta \backslash \alpha)] \\
\text { where }\left\{\begin{array}{l}
\beta=\arcsin (\mathbf{P} /(1+\mathbf{P})) \\
\alpha=\arcsin ((1+\mathbf{P}) / 2 \mathbf{P}) \\
\phi_{t}=\arcsin (1 / \mathbf{P})
\end{array}\right.
\end{gathered}
$$

and in which $\mathbf{K}(\alpha)$ is the complete elliptical integral of the first kind and $\mathbf{F}(\beta \backslash \alpha)$ is the incomplete elliptical integral of the second kind using the notation of Abramowicz and Stegun [Abramowicz and Stegun 1965]. The angle $\phi_{t}$ represents the rotation at the end of the spatula. As an alternative to this analytic procedure, equation (10) can be solved numerically for any particular value of the factor $P / E I$.

\section{EXPERIMENTAL VALIDATION and GECKO ADHESION}

Although it is hardly feasible to test the predictions of equations (7) (9) and (11) at the nano-scale of spatulae and seta, it is possible to demonstrate the mechanism suggested above 
at a much enhanced scale by substituting spring steel strip for the keratin spatula and small ceramic magnets to generate adhesion in place of van der Waal forces. With an appropriate choice of dimensions the numerical values of the relevant non-dimensional groups can be maintained in the same range as might be expected at the micro- and nano-scale. The experimental set-up is illustrated in the inset of Fig. 8. Spring steel strips of width $b$ $12.7 \mathrm{~mm}$, with thicknesses from $254 \mu \mathrm{m}$ to $635 \mu \mathrm{m}$ and lengths varying from ca. $50 \mathrm{~mm}$ to $120 \mathrm{~mm}$, were fixed at one end by small neodymium iron boron button magnets (MagnetExpert Part No: F200 10x10x5mm) to a steel plate which could translate freely on a linear ball bearing carriage. A vertical load was applied at the free end by mounting the assembly in a small tensile testing machine ( $\mathrm{JJ}$ model T5000). The free end of the strip was moved at a speed of ca. $20 \mathrm{~mm} / \mathrm{min}$ while the force was monitored until the situation became unstable and the elastic energy stored in the flexure of the steel strip was enough to break the magnetic bond. The non-dimensional quantities $\mathbf{P}$ and $\mathbf{L}$ could then be evaluated and plotted and so compared with the predictions of equations (8), (9) and (11) with the only fitting parameter being the initially unknown value of the 'adhesional energy' $w$.

Figure $7(\mathrm{a})$ shows the form of a steel $(E=210 \mathrm{GPa})$ cantilever with width $b=12.7 \mathrm{~mm}$, thickness $0.254 \mathrm{~mm}$ and length $100 \mathrm{~mm}$ subject to the loads indicated. Figure 7(b) illustrates the fact that the maximum root bending moment occurs when the tip load is just about $1 \mathrm{~N}$. For loads greater than this the reduction in moment arm more than compensates for the additional load and the root bending moment reduces. If the bending moment reaches the critical value $M_{0}$ then release will be initiated. If the applied moment fails to reach this value then eventually the steel strip slides over the face of the magnet as in steady-state peeling.

The experimental results from a number of different beams are shown in Fig. 8 over the range $0<\mathbf{L}<5$ and can be compared with the relation between $\mathbf{L}$ and $\mathbf{P}$ predicted by eqns. (8), (9) and (11). Two combinations of magnetic fixtures were used which corresponded to values of $w$ of 90 and $180 \mathrm{~J} \mathrm{~m}^{-2}$ respectively. When $\mathbf{L}<1$ the distinction between eqns. (8) and (9) are small but begin then to diverge. Eqn (9) is acceptable only up to values of $\mathbf{L} \sim 1.5$. Equations (8) and (11) take distinctly different values as $\mathbf{L}$ increases so that when $\mathbf{L}=5$ eqn (8) predicts $\mathbf{P}=0.4$ and eqn (11) $\mathbf{P}=1$. It is clear from the data that 
as the cantilever increases in slenderness the additional complexity of the elastica solution becomes more worthwhile.

Using typical dimensions, the operating point of a single gecko spatula can be plotted on the axes of Fig. 8. Take the length as $\ell=.8$ micron and the diameter of the shaft as $d=0.1$ micron then with $\quad E=2 \mathrm{GPa}, \quad E I=E \pi d^{4} / 64=9.8 \times 10^{-21} \mathrm{Nm}^{2}$. Taking $w=50 \times 10^{-3} \mathrm{Nm}^{-1}$, characteristic of organic surfaces, then $\quad \mathbf{L}=0.8$ and so, from Fig. 2 , $\mathbf{P} \approx 2$ which gives the force of adhesion $\quad P$ of each individual spatula as $P=2 \times .1 \times 10^{-6} \times 50 \times 10^{-3}=10 \mathrm{nN}$. This estimate is very similar to experimental measurements on single or a very small number of spatulae made in the AFM [Huber et al 2004]. This estimate of adhesive force might be increased if an allowance made for the broadening of the plate-like end of the spatula, i.e. $b>d$, but then equally a reduction would result from a decrease in the estimate of the effective work of adhesion $w$.

Because each seta is so very much stiffer than the large number of much smaller spatulae at its tip both the bulk of the deflection necessary to generate contact with the substrate and the greater part of the elastic energy of distortion are accommodated within the network of spatulae which constitute the 'adhesive' between the gecko toe structure and the underlying surface: in the terms of Kendall's adhesion classification [Kendall 1975b] this then represents a flexible joint where the greater part of the stored energy lies within the 'glue'. This mechanism of release is consistent with the observation that each gecko seta releases at a characteristic angle to a specific substrate when this corresponds to the generation of critical conditions within the spatulate adhesive [Hill et al 2011].

Equation (1), rewritten in the form of (12),

$$
P / b=w(1-\cos \theta)\left\{1-P^{2} / 2 b^{2} h w E\right\}
$$

demonstrates that for an adhesive of the same stickiness, i.e. value of $w$, the peel force for a tape of finite elasticity is somewhat less than that for a similar tape of effectively infinite stiffness i.e. for which $E \rightarrow \infty$; this reflects the fact that only a material of finite stiffness can store elastic strain energy which can subsequently contribute to the peeling process. If the tape is subject to an elastic strain as it applied to the substrate then further elastic energy can be stored and this too will be available to reduce the magnitude of the peel force $P$. This effect has been explored for conventional peel tests by a number of authors [Kaelble 1959, 
Williams and Kauzlarich 2004, Molinari and Ravichandran 2008] and has been invoked by Chen, Wu and Gao [2009] as a mechanism which could account for the rapidity of gecko spatula decohesion. It is presumed that this tensile energy is introduced into each spatula pad as it is dragged along the substrate surface during the attachment phase by a process of sequential stick-slip. However, it is generally much more efficient to absorb and store elastic deformation energy in either bending or twisting, i.e. as is done in conventional engineering springs, than directly in tension or compression. In addition, if the storage of energy depended principally on tensile stresses induced in the spatula pad by stick-slip sliding the efficiency of gecko motion would be more dependent on the nature of the substrate and its ability to cooperate in the generation of stick-slip than is usually observed. The mechanism suggested in section 2 which depends on elastic flexure rather than extension would seem to be more general.

\section{CONCLUSIONS}

Gecko setae/spatulae arrays adhere strongly to many surfaces and observation shows that the bond can be broken over many individual elements in a matter of millisecondsalmost as rapidly as it can be made. When the hierarchical structure of the gecko toe is loaded by either self-weight or during locomotion it will store mechanical energy in the elastic distortion of its constituent elements. Breaking the adhesive bond requires an energy input to which, it is suggested, this stored elastic energy can contribute so overcoming the effects of van der Waal (or indeed any other) forces that provide the gecko with its climbing ability. Because each seta is so very much stiffer than the large number of much smaller spatulae at its tip both the bulk of the deflection necessary to generate contact with the substrate and the greater part of the elastic energy of distortion are accommodated within the spatular structures.

\section{REFERENCES}

Abramowicz and Stegun I A (eds) Handbook of mathematical functions 1965 Dover Publications, New York

Autumn K, Dittmore A, Santos D, Spenko M and Cukovsky M 2006 J Exp. Biol. 209 3569

Autumn K, Hseih S T, Dudek D M, Chen J, Chitaphan C and Full R J 2006 J Exp Biol 209 260 
Autumn K, Liang Y A, Hsieh S, Zesch W, Chan W P, Kenny T W, Fearing R S and Full R J 2000 Nature $\mathbf{4 0 5} 681$

Autumn K, Majidi C, Groft RE, Dittmore A and Fearing R 2006 J Exp Biol 2093558

Bhushan B 2007 J Adh Sci and Technol. 211213

Chen B, Wu P and Gao H 2009 J R. Soc Interface 6529

Federle W 2006 J Exp. Biol. 2092611

Gao H, Wang X, Yao H, Gorb S and Arzt E 2005 Mechanics of Materials 37 275-85

Gillies A G, Henry A, Lin H, Ren A, Shiun K, Fearing R S and Full R J 2014 J Exp Biol 217283

Gravish N, Wilkinson M and Autumn K 2008 J R. Soc Interface 5(20) 339-348

Hill G C, Soto D R, Paettie A M, Full R J and Kenny T W 2011 J R Soc Interface 8926

Huber G, Gorb S N, Spolenak R and Arzt E 2004 Biol.Lett. 1 2-4

Israelachvili J N Intermolecular and surface forces 1992 Academic Press, London

Kaelble D H 1959 Trans. Soc. Rheol. 3161

Kendall K 1975a J Phys D Appl Phys 8512

Kendall K 1975b J Phys D Appl Phys 81449

Lee J, Bush B, Maboudian R and Fearing R S 2009 Langmuir 25(21) 12449-53

Love A E H 1927 A treatise on the mathematical theory of elasticity (4th Ed) CUP,

Cambridge

Majidi C, O'Reilly O M and Williams J A 2012 J Mech. Phys. Solids 60827

Molinari A and Ravichandran G 2008 J Adhesion 84961

Oyharcabal X and Frisch T 2005 Phys. Rev. E 7103611

Peattie A M, Majidi C, Corder A and Full R J 2007 J R Soc Interface 41071

Riedo E, Levy F and Brune H 2002 Phys. Rev. Lett. 88(18) 185505

Stewart M, McLachlan A D and Calladine C R 1987 Proc. Roy. Soc B 229381

Teodorescu M, Majidi C, Rahnejat H and Fearing R S 2008 STLE/ASME IJCT paper 71215

Tian Y, Pesika N, Zeng H, Rosenberg K, Zhao B, McGuiggan P, Autumn K and Israelachvili J 2006 PNAS 10319320

Vanhooydonck B, Andronescu A, Herrel A and Irschick D J 2005 Biol. J Linn. Soc 85 385

Varenberg M, Pugno N M and Gorb S N 2010 Soft Matter 63269

Williams J A and Kauzlarich J J 2004 J Adhesion 80433 


\section{Figure captions}

Fig. 1 Hierarchy of structures on the gecko toe. Adapted with permission from Gao H, Wang X, Yao H, Gorb S and Arzt E 2005 Mechanics of Materials 37 275-85 and Tian Y, Pesika N, Zeng H, Rosenberg K, Zhao B, McGuiggan P, Autumn K and Israelachvili J 2006 PNAS 10319320 (Copyright (2006) National Academy of Sciences, USA).

Fig. 2 (a) Geometry of steady-state peeling under action of peel force $P$. (b) The overall peeling force is linearly dependent on length of the peel line. Dividing the width $b$ of the adhered tape into two will make little difference to the total peel force. Adapted from Varenberg M, Pugno N M and Gorb S N 2010 Soft Matter 63269 with permission from The Royal Society of Chemistry.

Fig. 3 Total peel line as a function of body mass. Reproduced from Varenberg M, Pugno N M and Gorb S N 2010 Soft Matter 63269 with permission from The Royal Society of Chemistry.

Fig. 4 Sketches of attachment and detachment of a single seta by (a) approaching the substrate by rolling (or gripping) in (b) and rolling (or peeling) in (c). Both these actions will interact with energy stored as elastic deformation of the seta and its associated spatulae. Adapted, with permission from Tian Y, Pesika N, Zeng H, Rosenberg K, Zhao B, McGuiggan P, Autumn K and Israelachvili J 2006 PNAS 10319320 (Copyright (2006) National Academy of Sciences, USA).

Fig. 5 (a) Representation of a single seta loaded as in case (c) of Fig. 4. The seta has length $\ell_{2}$ and diameter $d_{2}$ and carries $n$ spatulae at its end each of which has dimensions $\ell_{1}$ and $d_{1}$. (b) Each spatula experiences a tip force $P$, which might be associated with adhesion between the spatula tip and the substrate, and a tip moment $M$, which arises because of the finite area of the flattened spatulate tip. The spatula bends as a cantilever through angle $\phi_{1}$. (c) The seta supports $n$ spatulae and deforms through angle $\phi_{2}$. In reality $\ell_{2}$ is more than 100 times greater than $\ell_{1}$ and $d_{2} / d_{1}$ is in excess of 5. There is elastic energy of distortion carried in both seta and spatulae.

Fig. 6 Idealised structural model of a single spatula structure from its point of attachment to the seta. Its shape can be described either in the cartesian coordinate axes $(x, y)$ or the intrinsic coordinates $(s, \phi)$. 
Fig. 7 (a) With increasing tip load the elastic deflection of the cantilever increases. (b) For the particular case of a steel cantilever length $100 \mathrm{~mm}$ with thickness $254 \mu \mathrm{m}$ and width $12.7 \mathrm{~mm}$ the moment at the root of the cantilever has a maximum when tip load is just over $1 \mathrm{~N}$.

Fig. 8 Experimental results plotted in non-dimensional form. Normalised load $\mathbf{P}=P / b w$ and length normalised $\mathbf{L}=\ell \sqrt{2 b w / E I}$. Inset illustrates the experimental arrangement. Open symbols $w=90 \mathrm{Jm}^{-2}$, closed $w=180 \mathrm{Jm}^{-2}$; squares thickness $.635 \mathrm{~mm}$, triangles $.406 \mathrm{~mm}$, circles $.254 \mathrm{~mm}$. 


\section{APPENDIX I}

The end rotation $\phi$ of an Euler-Bernoulli cantilever of length $L$ and uniform crosssection with second moment of area $I$ carrying a normal tip load $P$ can be written as $\phi=P L^{2} / 2 E I$ where $E$ is the Young's modulus of the beam material. The corresponding expression for the case in which $P$ is replaced by a tip moment $M$ is $\phi=M L / E I$. Thus, referring to Fig. 5, the tip rotation of an individual spatula, of which there are $n$, can be written as $\frac{P \ell_{1}^{2}}{2 E I_{1}}-\frac{\left(P \ell_{1}-M\right) \ell_{1}}{E I_{1}}$. Since for equilibrium $M=P\left(\ell_{1}+\ell_{2}\right)$ and if $\ell_{2} \gg \ell_{1}$ then the rotation $\phi_{\text {spatula }} \approx \frac{P \ell_{1} \ell_{2}}{E I_{1}}$. The total tip rotation $\phi_{\text {seta }}$ of the seta can be written as $\frac{n P \ell_{2}^{2}}{2 E I_{2}}$. If each beam is considered as end-to-end cylinders of radii $d_{1}$ and $d_{2}$ respectively, it follows that

$$
\frac{\phi_{\text {spatula }}}{\phi_{\text {seta }}}=\frac{P \ell_{1} \ell_{2}}{E I_{1}} \frac{2 E I_{2}}{n P \ell_{2}^{2}} \approx \frac{2}{n}\left(\frac{\ell_{1}}{\ell_{2}}\right)\left(\frac{d_{2}}{d_{1}}\right)^{4}
$$

The elastic strain energy $U$ stored in an Euler-Bernoulli beam of uniform crosssection with second moment of area $I$ and length $L$ can be written as

$$
U=\frac{2}{E I} \int_{x=0}^{L} M^{2} d x
$$

where $E$ Young's modulus, $M$ the bending moment and $x$ is measured along the beam axis. For a simple cantilever carrying and end load $P$ this provides the expression $U=P^{2} L^{3} / 6 E I$. When applied to the seta/ spatulae structure of Fig. 5 the energy within $n$ spatulae is $U_{\text {spatulae }}=\frac{n P^{2} \ell_{1}}{6 E I_{1}}\left(\ell_{1}^{2}+3 \ell_{2}^{2}\right)$ and within the seta $U_{\text {seta }}=n^{2} P^{2} \ell_{2}^{3} / 6 E I_{2}$. Since $\ell_{2} \gg \ell_{1}$ it follows that

$$
\frac{U_{\text {spatula }}}{U_{\text {seta }}} \approx \frac{3}{n}\left(\frac{\ell_{1}}{\ell_{2}}\right)\left(\frac{d_{2}}{d_{1}}\right)^{4}
$$




\section{ADHESIONAL INSTBILITIES and GECKO LOCOMOTION \\ John A Williams}

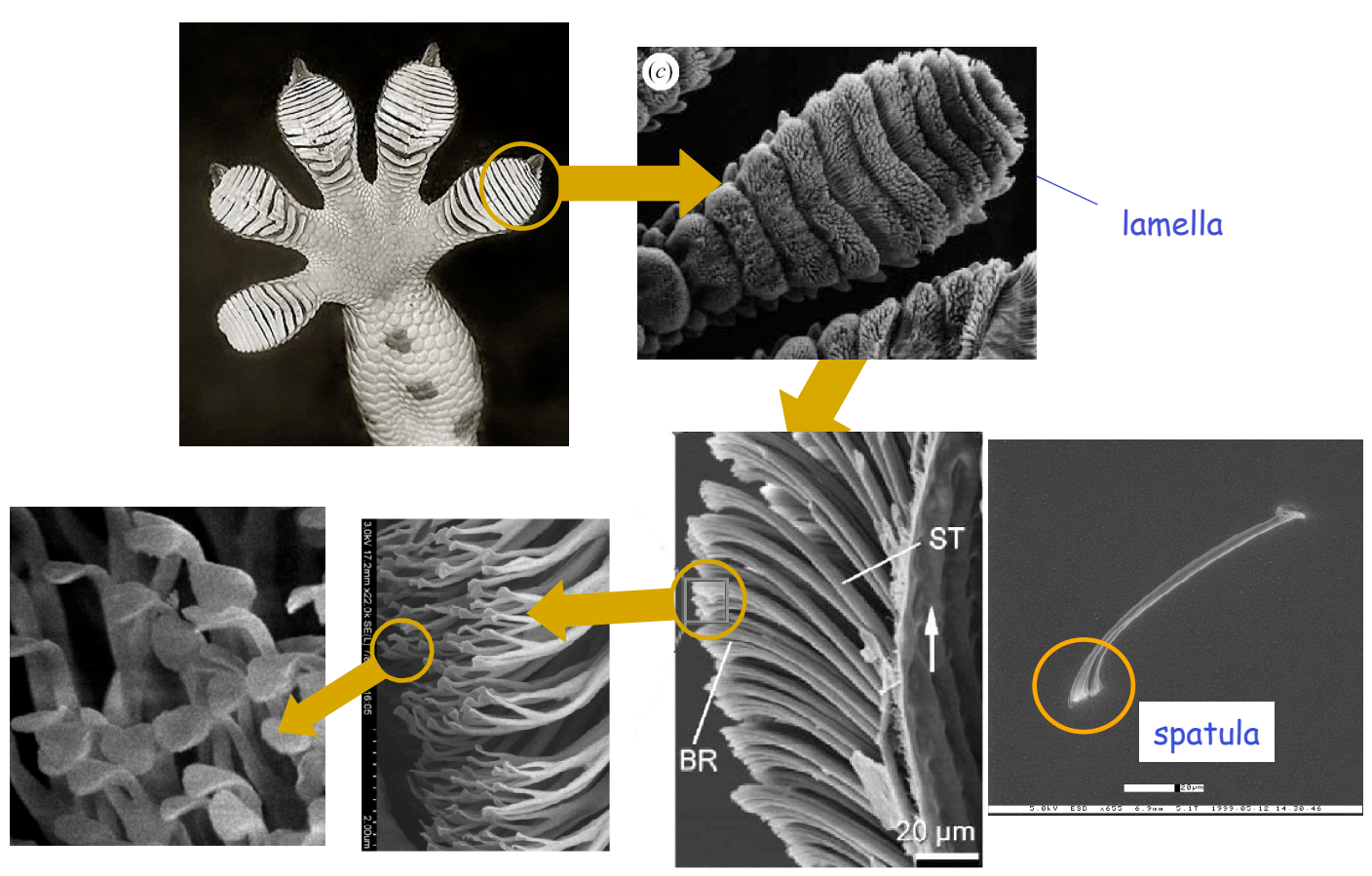

Fig. 1 Hierarchy of structures on the gecko toe. Adapted with permission from Gao H, Wang X, Yao H, Gorb S and Arzt E 2005 Mechanics of Materials 37 275-85 and Tian Y, Pesika N, Zeng H, Rosenberg K, Zhao B, McGuiggan P, Autumn K and Israelachvili J 2006 PNAS 10319320 (Copyright (2006) National Academy of Sciences, USA). 


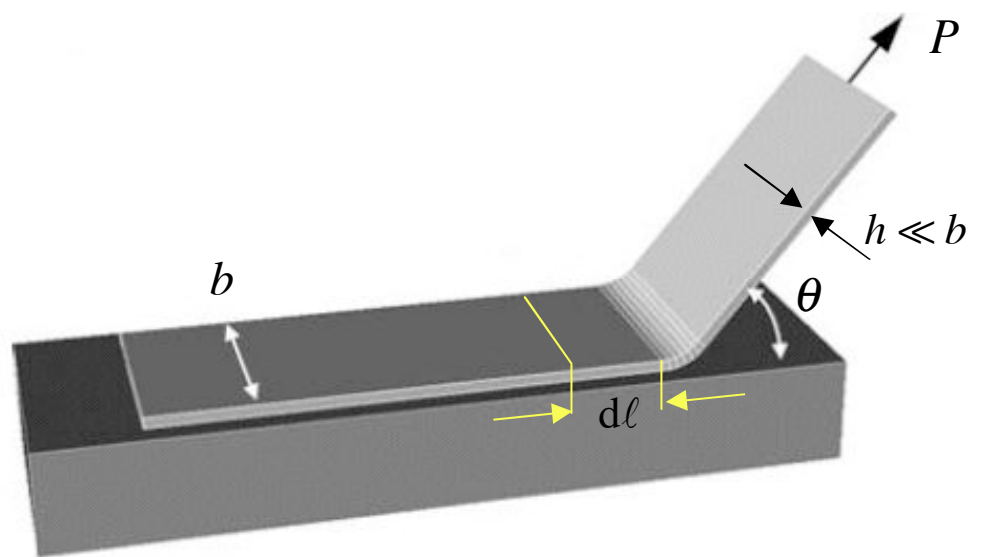

(a)

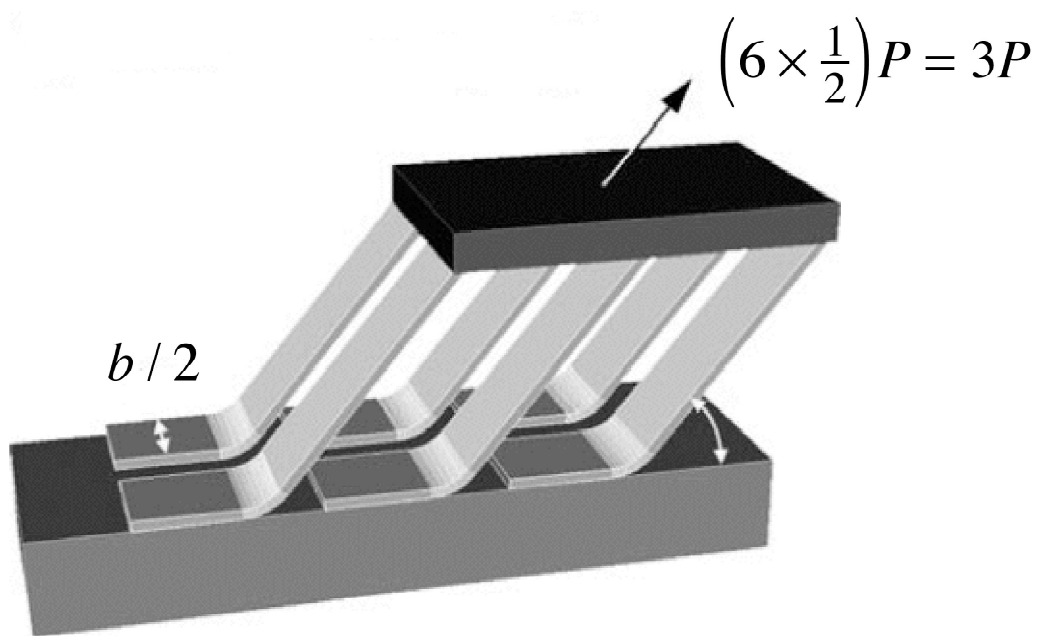

(b)

Fig. 2 (a) Geometry of steady-state peeling under action of peel force $P$. (b) The overall peeling force is linearly dependent on length of the peel line. Dividing the width $b$ of the adhered tape into two will make little difference to the total peel force. Adapted from Varenberg M, Pugno N M and Gorb S N 2010 Soft Matter 63269 with permission from The Royal Society of Chemistry. 


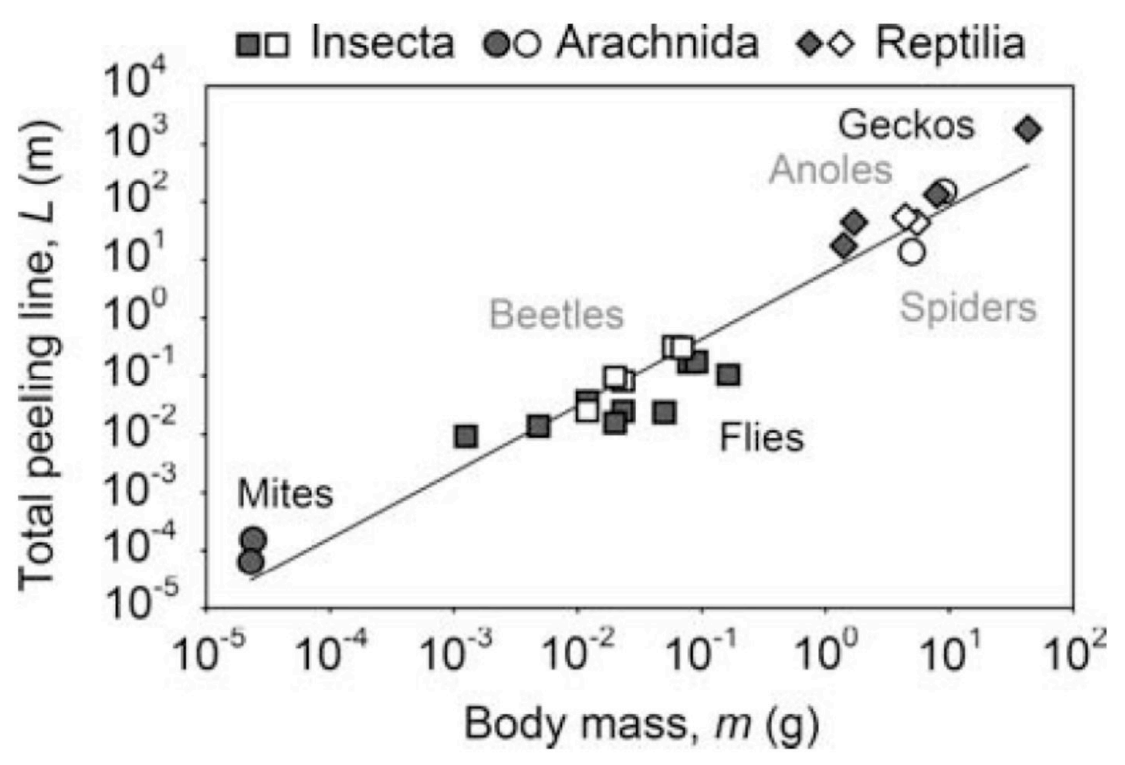

Fig. 3 Total peel line as a function of body mass. Reproduced from Varenberg M, Pugno N M and Gorb S N 2010 Soft Matter 63269 with permission from The Royal Society of Chemistry. 


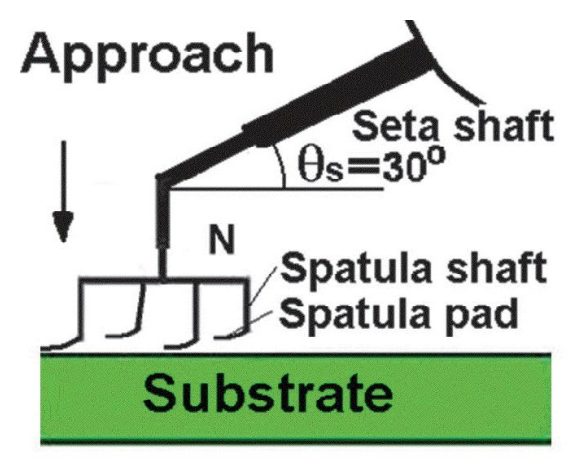

\section{b Rolling in Attachment}

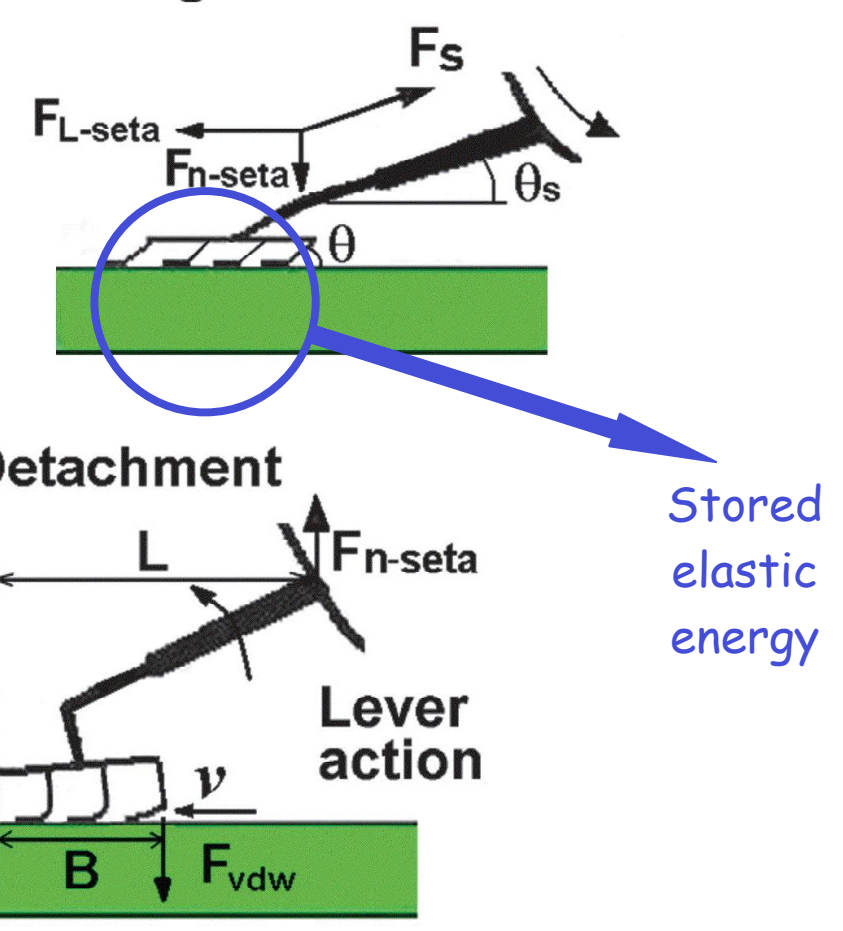

Fig. 4 Sketches of attachment and detachment of a single seta by (a) approaching the substrate by rolling (or gripping) in (b) and rolling (or peeling) in (c). Both these actions will interact with energy stored as elastic deformation of the seta and its associated spatulae. Adapted, with permission from Tian Y, Pesika N, Zeng H, Rosenberg K, Zhao B, McGuiggan P, Autumn K and Israelachvili J 2006 PNAS 10319320 (Copyright (2006) National Academy of Sciences, USA). 


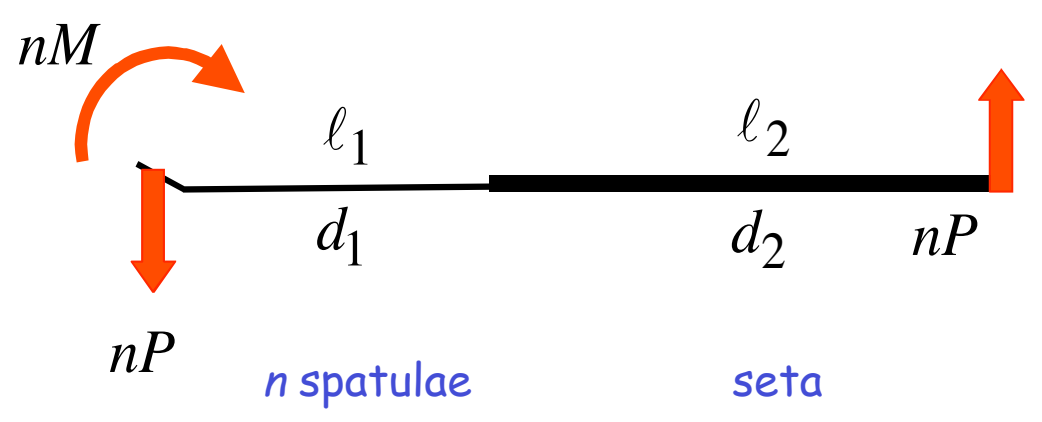

(a)

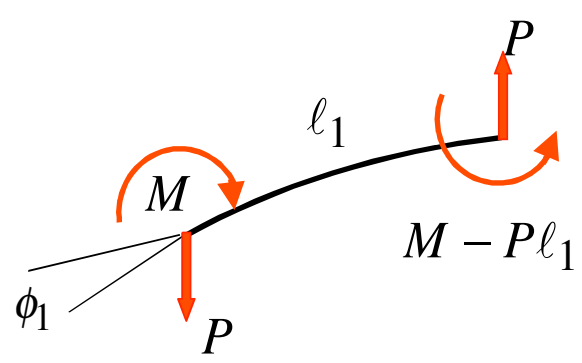

single spatula

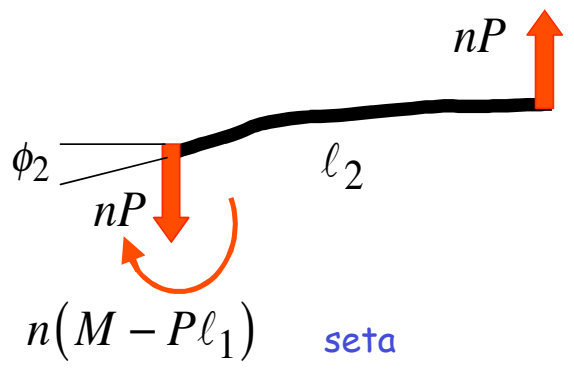

(b)

(c)

Fig. 5 (a) Representation of a single seta loaded as in case (c) of Fig. 4. The seta has length $\ell_{2}$ and diameter $d_{2}$ and carries $n$ spatulae at its end each of which has dimensions $\ell_{1}$ and $d_{1}$. (b) Each spatula experiences a tip force $P$, which might be associated with adhesion between the spatula tip and the substrate, and a tip moment $M$, which arises because of the finite area of the flattened spatulate tip. The spatula bends as a cantilever through angle $\phi_{1}$. (c) The seta supports $n$ spatulae and deforms through angle $\phi_{2}$. In reality $\ell_{2}$ is more than 100 times greater than $\ell_{1}$ and $d_{2} / d_{1}$ is in excess of 5. There is elastic energy of distortion carried in both seta and spatulae. 


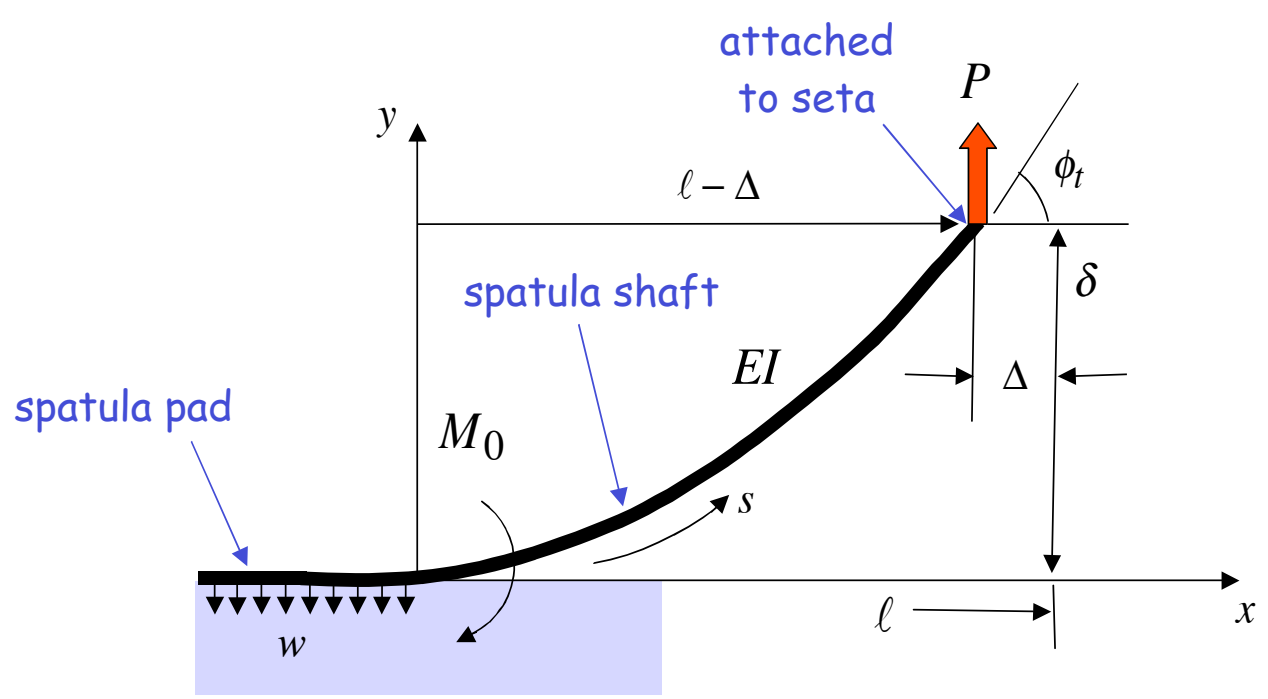

Fig. 6 Idealised structural model of a single spatula structure from its point of attachment to the seta. Its shape can be described either in the cartesian coordinate axes $(x, y)$ or the intrinsic coordinates $(s, \phi)$. 


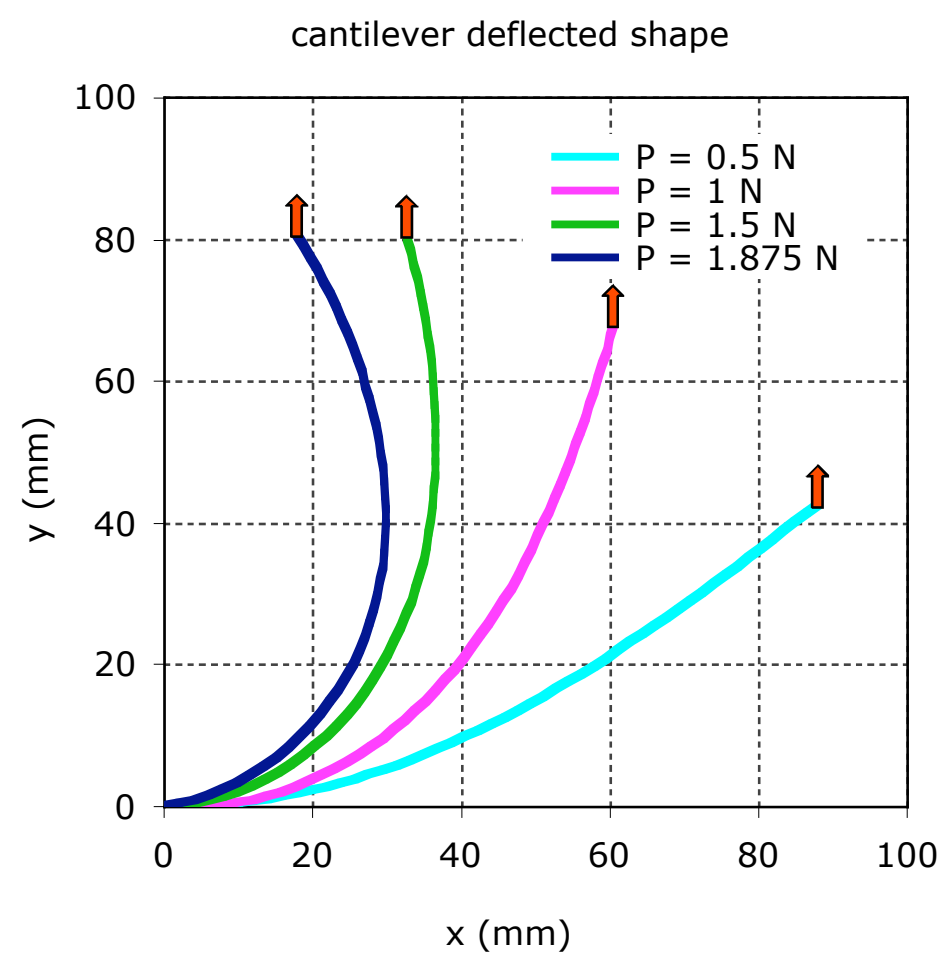

(a)

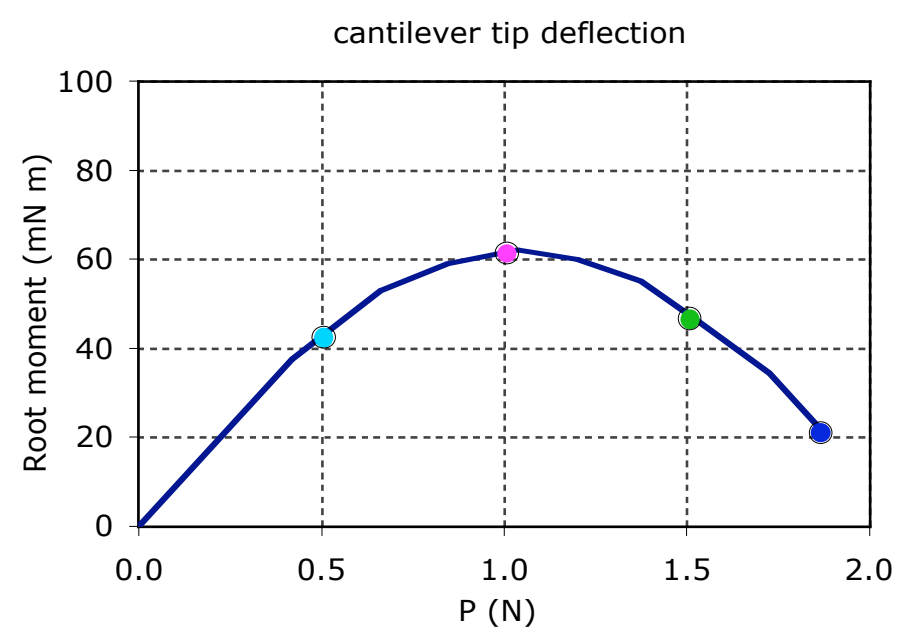

(b)

Fig. 7 (a) With increasing tip load the elastic deflection of the cantilever increases.

(b) For the particular case of a steel cantilever length $100 \mathrm{~mm}$ with thickness $254 \mu \mathrm{m}$ and width $12.7 \mathrm{~mm}$ the moment at the root of the cantilever has a maximum when tip load is just over $1 \mathrm{~N}$. 


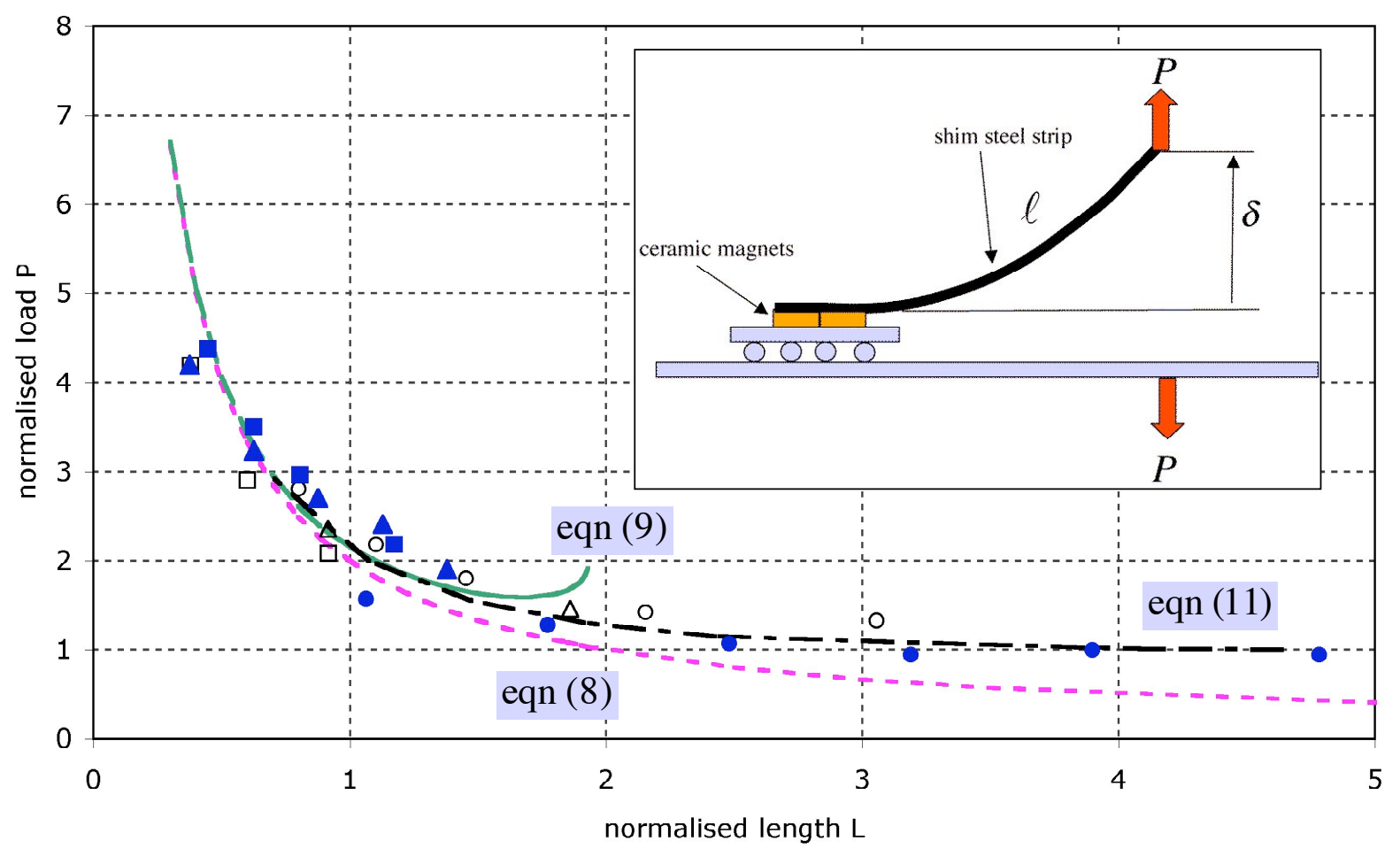

Fig. 8 Experimental results plotted in non-dimensional form. Normalised load $\mathbf{P}=P / b w$ and length normalised $\mathbf{L}=\ell \sqrt{2 b w / E I}$. Inset illustrates the experimental arrangement. Open symbols $w=90 \mathrm{Jm}^{-2}$, closed $w=180 \mathrm{Jm}^{-2}$; squares thickness $.635 \mathrm{~mm}$, triangles $.406 \mathrm{~mm}$, circles $.254 \mathrm{~mm}$. 